\title{
Project "Development of the Methodology of Experiment and Technical Support for Studies of the Flow Cyclotron Maser in the Earth's Magnetosphere by Creating an Artificial Ionization Cloud From a Geophysical Rocket"
}

\author{
S. Chernouss $\left(1,{ }^{*}\right)$, V. Alpatov(2), A. Demekhov(3), C. Deehr( ${ }^{(4)}$, U. Brandstrom(5), O. Widell(6),
} N. Ivchenko(7), A. Koustov(8), R. Pirjola(9), T. Sergienko(5), I. Sandahl(5,†), G. Marklund(7)

1. Polar Geophysical Institute KSC RAS, Apatity Murmansk region, Russia.

2. Institute of Applied Geophysics, Moscow, Russia.

3. Institute of Applied Physics RAS, Nizhny Novgorod, Russia.

3. Institute of Applied Geophysics, Moscow, Russia.

4. Geophysical Institute University of Alaska, Fairbanks, USA.

5. Institute of Space Physics, Kiruna, Sweden.

6. ESRANGE, Kiruna, Sweden.

7. Royal Institute of Technology, Stockholm, Sweden.

8. University of Saskatchewan, Saskatoon, Canada.

9. Finnish Meteorological Institute, Helsinki, Finland.

${ }^{(*)}$ Email: chernouss@pgia.ru

Recibido / Received: 30/01/2011. Aceptado / Accepted: 30/08/2011.

\begin{abstract}
:
Investigation of the wave particle interaction in the magnetosphere and ionosphere by controllable experiment in near Earth space is in focus of modern space geophysics. We propose to stimulate auroral precipitation by changing parameters of the Flow Cyclotron Maser (FCM) and test the FCM model itself. One of the main goals of the project is inducing of artificial pulsating aurora.
\end{abstract}

Keywords: Ozone, UV Radiation, Multichannel Radiometer.

\section{REFERENCIAS Y ENLACES / REFERENCES AND LINKS}

[1]. C. Deehr, G. Romick, "Pulsating aurora induced by upper atmosphere barium release", Nature 26, 177-179 (1977).

[2]. V. Yu. Gaydukov, M. G. Deminov, Yu. V.Dumin, A. N. Omelchenko, Yu. A. Romanovsky, V. M. Feygin, "The generation of electric fields and particle fluxes by plasma injection in the high latitude ionosphere", Kosmicheskie Issledovania 31, 54-62 (1993).

[3]. V. Yu. Trakhtengerts, S. A. Chernouss, V. R. Tagirov, "A circulating cyclotron maser and pulsed VLF emissions", Geomagn. Aeron 26, 77-82 (1986).

[4]. A. G. Demekhov, V. Yu. Trakhtengerts, "A mechanism of formation of pulsating aurorae", J. Geophys. Res. 99, 5831-5841 (1994).

[5]. http://www.istc.ru/istc/db/projects.nsf/

[6]. S. A. Chernouss, V. Yu. Trakhtengerts, V. Alpatov, C. Deehr, U. Brandstrom, O. Widell, "Artificial pulsating aurora project: stimulation of auroral precipitations by barium release from a rocket", Proceedings of $18^{\text {th }}$ European Rocket and Balloon Programmes and Related Research, pp. 431-436, Visby, Sweden, European Space Agency (2007).

[7]. G. Marklund, N. Brenning, G. Holmgren, G. Haerendell, "On transient electric fields observed in chemical release experiment by rockets", J. Geophys. Res. 92, 4590-4600 (1987).

[8]. http://www.iki.rssi.ru/resonance 


\section{Introduction}

Existing experimental basis is observations of pulsating aurora in Alaska [1] and pulsating particle precipitation in Atlantic Ocean [2] in the rocket experiments, which were carried out by Americans in the Poker Flat Range and Russians by the research ship "Professor Zubov". The auroral pulsations were apparently triggered after the artificial release of Ba clouds into the ionosphere. Results of these experiments were published [1,2], but without any acceptable theory.

This theory was developed independently $[3,4]$ and based on a magnetic flux tube containing a filament of enhanced plasma concentration and energetic electrons with anisotropic velocity distribution. These particles enter the FCM in the course of magnetic drift. There they switch on the cyclotron instability and emerge from the filament with isotropic velocity distribution. The model was constructed on the basis of simultaneous observations of aurora and natural very low frequency (VLF) emissions and quantitatively explains pulsating energetic particle precipitation and auroral pulsations, which are correlated with pulsating VLF emissions. The essential feature of FCM is a strong dependence of the reflection coefficient of the whistler mode on the ionization at the foot point of the filament in the ionosphere. This dependence gives us the possibility to generate the auroral pulsations by creating an ion-cloud mirror in the ionosphere. Thus, we have unexplained experimental data and general theory of FCM, which can be verified by new experiments now.

\section{Project summary}

The main objective of the project is to develop a methodology of an experiment on the direct verification of flow cyclotron maser (FCM) in the magnetosphere and a study of its properties by chemical release into the upper atmosphere from a geophysical rocket. The International Science and Technology Centre (ISTC) scientifically supports this project. Short title of the project is "Flow cyclotron maser in the Earth magnetosphere" Some general features of the project can be found at the ISTC site [5] and in [6]. One of the main goals of the project is stimulating artificial aurora in the polar ionosphere. Project objectives are the next: development of the methods and models for calculation and prediction of the FCM parameters and indicators of its operation during the experiment; development of the methodology of an experiment, methods and models for detection and recording of the FCM
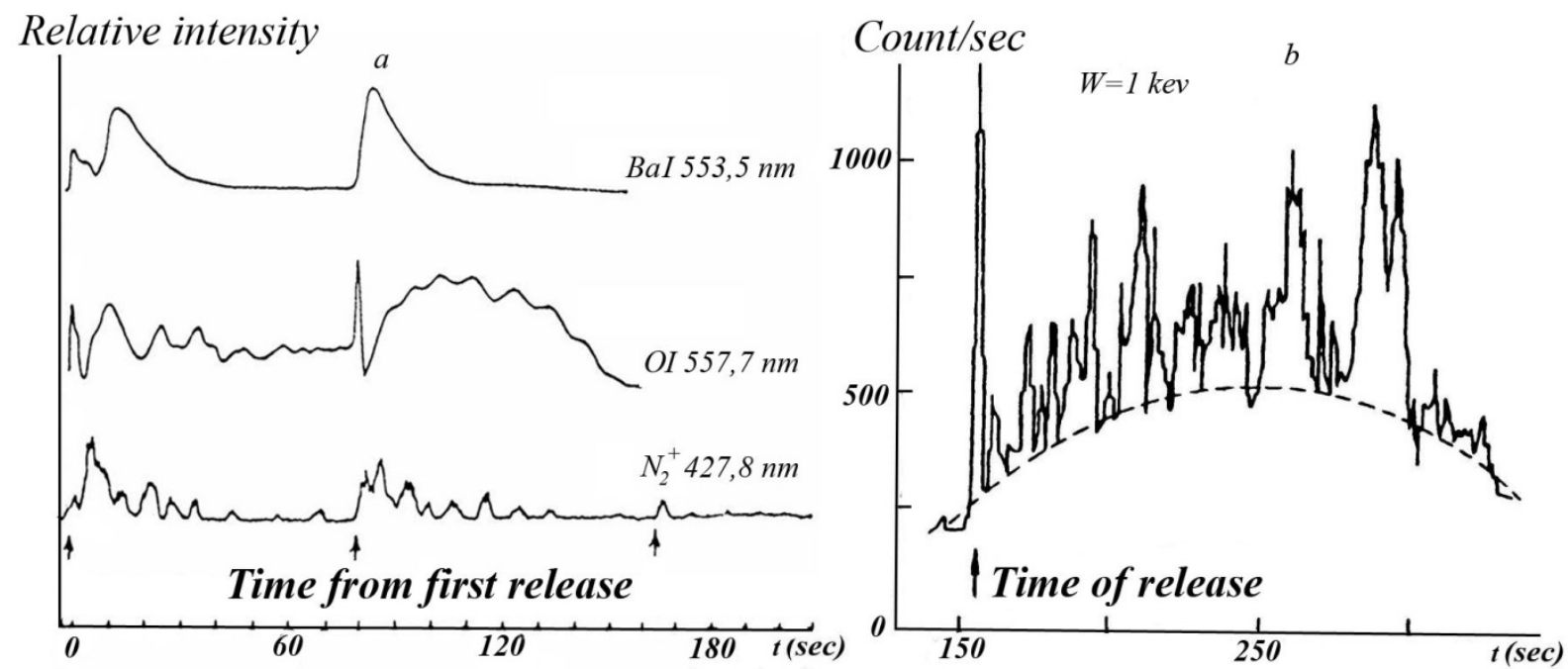

Fig. 1. (a) Observations by a TV spectrograph of two auroral emissions and one neutral Ba emission associated with upper atmospheric Ba releases made from Poker Flat, Alaska. The times of three releases are indicated by arrows. b) Electron count variations during "Auroral Trigger" experiment with Ba - release in the North Atlantic $\left(67.78^{\circ} N, 0.025 \mathrm{~W}, L=6.15\right)$ measured by the rocket counter. 


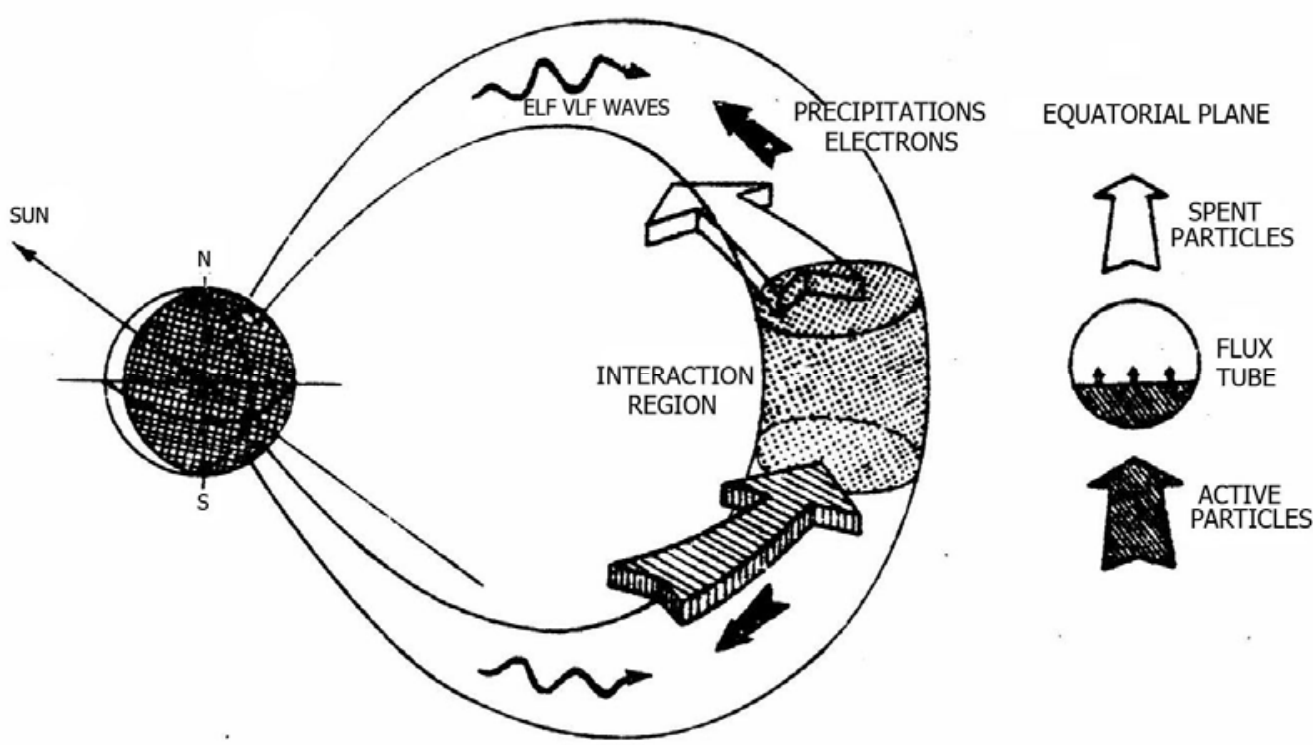

Fig. 2. Scheme of Flow Cyclotron Maser in the magnetosphere and equatorial cross-section of duct with a cold plasma..

indicators; development of an empirical model of short-term (hours) forecasting the heliogeophysical situation and weather conditions for carrying out the rocket experiment and optimizing the pre-launch preparation of the rocket site; development of an on-board injector for artificial cloud release; fitting technical parameters and characteristics of the on-board injector (generator) with the parameters and characteristics of the control and datatransmission systems of a geophysical rocket used at ESRANGE site (Sweden); development of the methodology and special equipment for ground-based observations of artificial clouds, natural and artificial auroras in the ionosphere, and low frequency emissions; modification and testing of ground-based (TV, photometer, spectral, interferometer, radio tomography) diagnostic equipment for the observations of FCM specific characteristics during the experiment, under the natural (background) conditions.

\section{Project narrative}

Within the framework of the project, the methodology of an experiment and technical facilities (including production of the brassboard models of the on-board equipment and groundbased diagnostic equipment) will be developed for studies of a flow cyclotron maser (FCM) in the Earth's magnetosphere with use of creation of artificial ionized cloud released by a geophysical rocket. During the investigation of the self-consistent theory of radiation belts a group of the project participants developed the conception of the flow cyclotron maser (FCM) in the Earth's magnetosphere, in which energetic charged particles represent active substance, while magnetic force tubes, filled with cold plasma and limited by the ionospheres of both hemispheres serve as a resonator. The main confirmation of FCM presence in the Earth's magnetosphere is the development of pulsating forms of auroras and related to them low frequency emissions of chorus type, observed during natural magnetosphere disturbances. All this is necessary, however not enough to build an accurate quantitative model of interaction of low frequency waves and particles within the FCM theory. Therefore, it is proposed to prepare a controlled experiment, consisting of creation of an artificial ionized cloud (ionosphere mirror), which can significantly increase the reflection coefficient of low frequency waves from the ionosphere and thus excite electron precipitation from the magnetosphere into the ionosphere and cause artificial pulsating auroras. The authors of the project and collaborators yet prior to the development of the FCM theory first found some indicators in rocket experiments, which were the evidence of high probability of real functioning of FCM in the 
magnetosphere of the Earth (detection of pulsating precipitations of particles and modification of auroras during formation of ionized barium clouds in the upper atmosphere), however, these works had been carried out without knowledge about simultaneous detection of low frequency emissions, by which one could reliably judge about the cause of the stimulated precipitations and artificial auroras. Within the framework of the project, it is planned to produce brassboard models of the on-board rocket complex for cesium injection, to fit the technical characteristics of a rocket and payload to each other, to develop new and to modify existing ground-based optical, radiophysical and geophysical measurement tools, to develop a methodology of prediction and optimal choice of the quantity of released substance, time and height of injection on the basis of estimating the possible geophysical situation. In this project it is intended to carry out experimental observations (imitation experiments) with use of the developed groundbased diagnostic equipment to determine the background (without chemical injection) dynamics of the parameters of natural pulsating auroras and accompanying geophysical effects.

\section{Scheme of the experiment and equipment}

The Cs instead of the Ba as substance [7] was selected for injection after our complex analysis of the problem. Northern Sweden is in auroral zone and rather good place for the experiment on the induction of pulsating aurora by chemical release into the polar ionosphere. There are ESRANGE with rockets launch facilities and IRF network of low light CCD cameras, which permit recording natural and artificial weak images of auroral effects in the upper atmosphere. PGI, ESRANGE and IRF devices can provide the spectral observations. There are also satellite station for weather forecast and network of magnetometers and riometers of ESRANGE and IRF, which can be used for real time control of the current geophysical situation and selecting the launch time. There are two necessary conditions for the time of launch. Heights of the ionized cloud release (about $180 \mathrm{~km}$ ) most probably must be in sunlit conditions and typical recovery phase of the substorm should be existed near the zenith of the launch station. A payload consisting of canister with chemical components and the operation system for an explosion can be prepared by Institute of Applied Geophysics, which has a great experience of this kind work. VLF observations could be provided by PGI and maybe other invited participants. The Alaska Geophysical Institute team can provide long-term forecasting. All theoretical and practical questions concerning the experiment, selection of carrier, assembly, integration and test of payload equipment are discussed and detailed by partners now and presented in the report. The project could be realized simultaneously with the international satellite project 'Resonance" [8], which turned to the same problem of interaction of electromagnetic waves and particles in magnetospheric and ionospheric plasma.

\section{Concluding remarks}

The main results of the project should be a new fundamental knowledge of energetic particle states in the radiation belts and their dynamics at interaction with the electromagnetic waves and new physical mechanisms acting in the near Earth space. Theoretical and experimental developments include methods and models of calculation and prediction of the conditions for active physical experiment for verification of a FCM model, the brassboard models of the onboard generators for cesium injection into upper atmosphere, ground-based optical, radio physical and geophysical equipment adapted for the conditions of a supposed active experiment as well as for different geophysical background observations and experiments. Containers for keeping chemical components and onboard system of injection control, which are at the disposal of the project authors, will be used for the purpose of research work of international teams. This work will be of fundamental importance for physical investigation of plasma processes in laboratory and space plasmas and for the diagnosis of energetic particles in radiation belts and their dynamics upon the interactions with electromagnetic waves. An important line of applied studies in this project 
is the preparation active experiments under optimal and monitored conditions. This experience can be employed in future for various applied experiments in the near-Earth space. Current status of the project in the ISTC: it completely went through scientific expertise and was approved for the time being without necessary financial support.

\footnotetext{
${ }^{\dagger}$ Ingrid Sandahl sadly passed away in May 2011
}

\section{Acknowledgements}

Some of authors thank the ISTC for the financial support by the Mobility Grant for providing of presentation of this project \# 3915 ISTC at the 37 European Meeting on Atmospheric Studies by Optical methods in Vallidolid (Spain) and support of the current work on the project by the RFBR grant No.10-05-00792-a. 